\title{
Do Social Robots Walk or Roll?
}

\author{
Selene Chew ${ }^{1,2}$, Willie Tay ${ }^{1,2}$, Danielle Smit ${ }^{1}$, and Christoph Bartneck ${ }^{1}$ \\ 1 Eindhoven University of Technology \\ Department of Industrial Design \\ Den Dolech 2, 5600MB Eindhoven, The Netherlands \\ d.h.smit@student.tue.nl, c.bartneck@tue.nl \\ 2 National University of Singapore \\ Department of Architecture, Design and Environment \\ Industrial Design \\ 4 Architecture Drive, Singapore 117566 \\ sel.chew@nus.edu.sg, willie.tay@nus.edu.sg
}

\begin{abstract}
There is a growing trend of social robots to move into the human environment. This research is set up to find the trends within social robotic designs. A sample of social robotic designs is drawn to investigate on whether there are more legged social robots than social robots with wheeled. In addition we investigate whether social robots use legs or wheels for locomotion, and which continent produces the most social robotic designs. The the results show that there are more legged robots, most robots use them for locomotion and Asia is the continent that produces most social robots. It can be concluded that there is a trend that social robots are more and more designed to have legs instead of wheels. Asia has more different social robotic designs to cater to different needs of human.
\end{abstract}

Keywords: social robots, leg, wheel, locomotion, continents.

\section{Introduction}

Social robots are playing an important part in our future society. Already today, service robots outnumber industrial robots [8]. They are intended to entertain, educate and care for their users. One of the main design questions for these types of robots is if they should walk or roll. Walking robots can operate easier in an environment that is originally designed for human beings. Stairs, steps and the variety of floor coverings do not bother them too much. However, rolling is a much more reliable form of locomotion since the robots do not need to keep their balance. Since the field of social robotics has been confronted with this problem for some time, it appears useful to investigate how the robotic developers have overcome it.

A social robot is an autonomous robot that interacts and communicates with humans or other autonomous physical agents by following social behaviours and rules attached to its role 2. This definition suggests that a social robot must have a physical embodiment. We followed this definition and did not include 
screen based agents, industrial robots or military robots. Surveys about the field of social robotics date back to already 2001 [1], meaning that first robots can already be expected to appear 15 years ago. We therefore included robots that were at least available since 1995.

It is very difficult to collect information about the absolute number of social robots that were released. Arguably, Aibo was the most sold social robot so far with 140.000 units sold [4. We therefore focused not on the absolute number of robots, but on the count of different robotic designs. We did not require the social robot to be commercially available in order to be considered for this survey, since many social robots never left the research laboratories. It is sufficient if only one example robot was ever built. Thus to identify the trends of social robots, the following questions formed the focus of the research:

1. Are there more social robotic designs with legs or wheels?

2. Are the legs or wheels of the social robots used for locomotion?

3. Which continents develop more social robots?

\section{Method}

\subsection{Data Gathering}

A sample size of 106 social robots was randomly gathered through a web search. Only general keywords like "Robots" and "Social Robots" were used as search terms in different search engines, such as Bing, Google and Yahoo. Specific keywords like: "robots + legs", "humanoid + robots" or "wheels + robots" were not used, since they would have biased the sample of the search results. We assume that the 106 found social robots represent a random sample of social robots. We then manually inspected the robotic designs and recorded their features in a spreadsheet. The following features were extracted:

- Ability to perform locomotion

- If the robot has legs and how many

- If the robot has wheels and how many

- Country origin of the robot

- Creator of the robot

- Web or book references

The robots that were included in the analyses are listed in table 1 .

\subsection{Categorization}

Based on the collected raw data, the robots are categorized into four categories: With Legs only, With Wheels only, With Both or With None. Their definitions as follows:

Legs. Any limbs that have contact with the ground regardless of ability to perform locomotion. Example: Asimo 6] 
Table 1. Robots included in the analyses

\begin{tabular}{|c|c|c|}
\hline Actroid DER 2 & i-Qbot & Rave Raffe \\
\hline Aibo & iCat & Reddy \\
\hline Aida & $\mathrm{iCub}$ & reem-A \\
\hline AirJelly & Irobot & Reem-B \\
\hline ApriPoko & IsaacRobot & Ri-Jo \\
\hline ASIMO & Joe Robot & RIBA \\
\hline Autom & Joebot & Robin \\
\hline BigDog & Kande and Turin & Roborover \\
\hline Callo & KASPAR & Robosapien \\
\hline CB2 & Keepon & Robosapien V2 \\
\hline CHARLI & Keiko & Robot Cub \\
\hline coco & KHR-1 & Robot Jockey \\
\hline Doki & Kismet & Robovie-IV \\
\hline doppelganger robot & Kiyomori & Rovio \\
\hline Dream Parrot & Kobian & Rubot \\
\hline Dyson Robotic Vacuum Cleaner & Konan & Serviboy \\
\hline emuu & Kozirou and Kotarou & Sessha and Soregashi \\
\hline ERS-311 & Land Walker & Spykee \\
\hline EveR1 & Leonardo & Steam Walker \\
\hline EveR2-Muse & Liangliang & T-Bot \\
\hline evolta & Maggie & $\mathrm{T} 2-5$ \\
\hline Femisapien & Mahru & Tamanoi Vinegar Robot \\
\hline flame & Mahru-R & Tiro \\
\hline Flexible Spine Robot & Mahru-Z & Tofu \\
\hline Flexible-jointed Robot & MANOI-AT01 & Topio \\
\hline G-Dog & MANOI-PF01 & Toyota's Robot Quartet Band \\
\hline Germinoid & Miss Repliee Q1 & Toyota's Running Humanoid Robot \\
\hline Gil and Gil Son tan & Mr. Personality & Tri-Bot \\
\hline 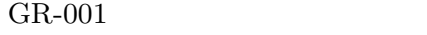 & nao & Twendy-One \\
\hline guroo & Nexi & USB robot webcam \\
\hline gymnast robot & Paro & WABIAN-2R \\
\hline HOAP-3 & Plen & WE-4RII \\
\hline HRP-2 & Рopo & Wine-Bot \\
\hline $\mathrm{HRP}-4 \mathrm{C}$ & Probo & Wrex The Dawg \\
\hline Huggable & QRIO & Zeno \\
\hline I-FAIRY Robot & & \\
\hline
\end{tabular}

Wheels. Appears to have a circular frame or disk arranged to revolve on an axis regardless of ability to perform locomotion. Example: Papero [5]

Both. With properties of Legs and Wheels regardless of the ability to perform locomotion

None. Has neither the properties of legs and wheels regardless of ability to perform locomotion. Example: Paro[7]

In addition, the robots are also categorized into their ability to perform locomotion, which means the act or power of moving from place to place. This is to find the relation between the use of legs or wheels for locomotion. An example of a robot that has human like legs yet unable to walk is Geminoid HR1 [3]. Based 
on the country origin of the social robots, they are also categorized into their respective continents based on the following definitions:

America. Consist of 35 sovereign states (North \& South America)

Europe. Consist of 47 sovereign states (Northern, Eastern, Southern and Western Europe)

Asia. Consist of 47 sovereign states (North, South, East, West, Central Asia and South East Asia)

Others. Any countries that does not fall in any of the three continent mention about (Asia, America \& Europe)

As there were only two out of hundred and six robot designs that were made in Australia or Africa, thats why the focus was set on comparing the three major continents of robot creators, and labeling the rest as "others".

\section{Results}

\subsection{Robots with Legs or Wheels}

From the sample of 106 robots, 63 robots had only legs, which covered about $59 \%$ of the total robots (see Figure 1). To determine whether there are more robots with legs or wheels, the number of robots with legs only and wheels only are compared.

Based on the data, there are 20 social robotic designs with wheels, while the other 63 robotics designs are with legs. We calculated a $99 \%$ confidence interval around the sample proportion for the legged robots of $\frac{63}{83}=0.759$ : Pro $[0.638$

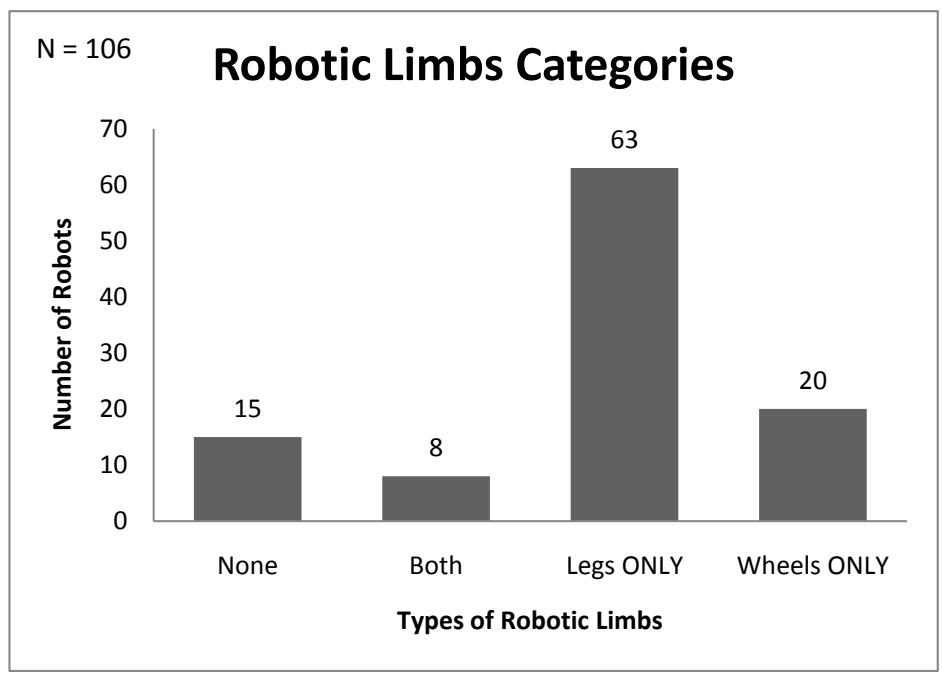

Fig. 1. Number of robots with different robotic limbs 
$<\Pi<0.880]=0.99$. The confidence interval does not include the value of 0.5 and we can therefore conclude with $99 \%$ certainty that there are significantly more robotic designs with legs than with wheels.

We also collected information about the number of legs and wheels per robot. The mean number of legs or wheels is 2.2 and 3.21 respectively (see Figure 2). It is thus more common for a robot with legs to have two legs. This shows that social robots with legs are likely to be made with two to imitate the action of human walking. On the other hand, robots with wheels are likely to have 3 wheels: two for movement and one for balance.

\subsection{Ability of Robots to Perform Locomotion}

The relationship between the ability of the robot to perform locomotion and whether it has either legs or wheels to perform is determined in Figure 3, which shows that 78 out of 91 of the robots with either legs or wheels can use their legs/wheels to perform locomotion.
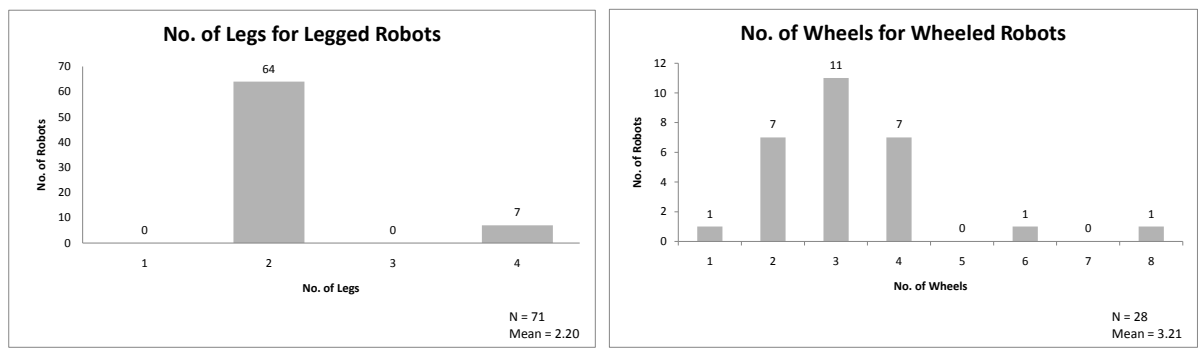

Fig. 2. Count of number of wheels or legs per robot

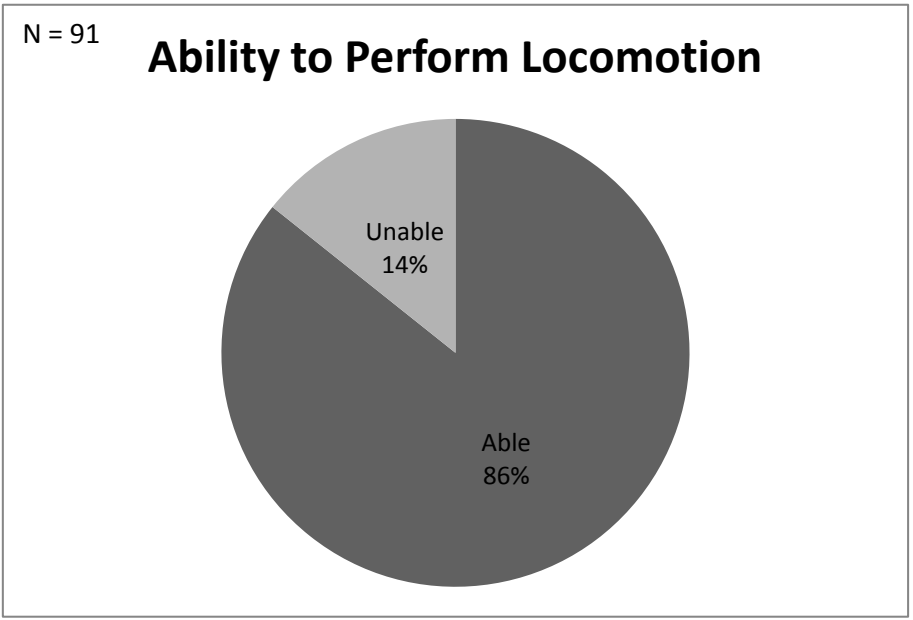

Fig. 3. Proportion of robots with legs or wheels that can perform locomotion 
Based on the data, we constructed a $95 \%$ confidence interval around the sample proportion of $\frac{78}{91}=0.857: \operatorname{Pro}[0.785<\Pi<0.929]=0.99$. Since 0.5 is not within the confidence interval, we can conclude that there are significantly more social robots that use locomotion than robots that do not.

\subsection{Geographic Distribution}

The pie-chart below shows an overview of which continent contributes the most different robot designs (see Figure 4). The chart shows that, Asia covers 55\% of the whole sample. This is largely contributed by one of the worlds top robot creator, Japan. Japan alone covers $42 \%$ of the total sample, Korea follows with 10\%. With Japan and Korea in the top three, Asia may continue to hold the status of being the top robot-creating continent.

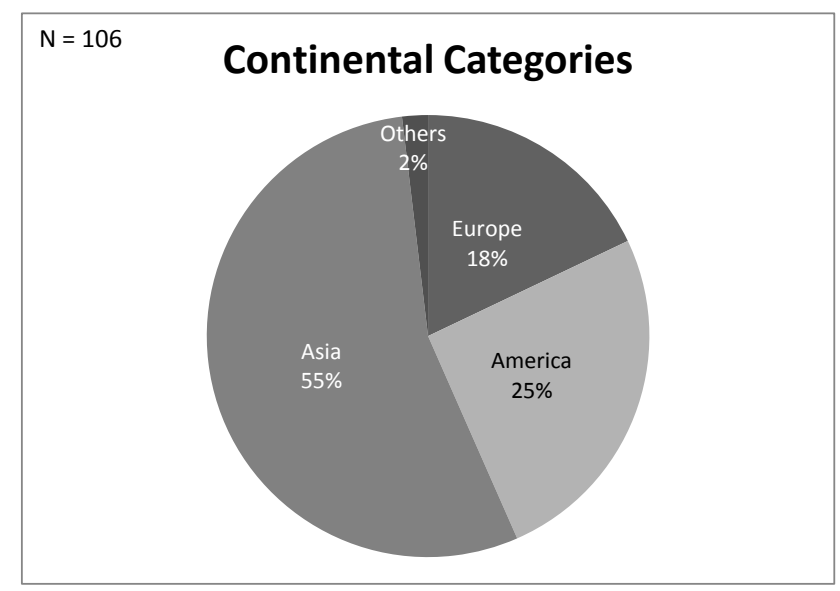

Fig. 4. Proportion of robotic designs based on their continent origin

\section{Conclusions}

When it comes to deciding whether to use legs or wheels for social robots, each of them definitely has strong advantages of their own. Wheels are more stable and balance in terms of ability to perform locomotion and operation. However, social robots are supposed to be made to interact with humans. This would mean that they are operating in an environment designed for humans with legs. Thus legged robots have the advantage of ease of moving in and interacting with the human environment. From the data, there is a $99 \%$ certainty that there are more than $50 \%$ of robots with legs. We can also be $95 \%$ sure that if the robots have either legs or wheels or both, most of them can perform locomotion. So the limbs or wheels are less likely to be for aesthetical purpose, but also used for locomotion. From these fact mentioned above, there is a trend that social 
robots tend to have legs that can locomotion. So it is likely that social robots in future will be built with legs in order to integrate into the human environment. It also shows that interaction with humans is no longer the only function of social robots; they must also have the ability to perform locomotion in a environment based on human movement.

This study also has some limitations. We were not able to reliably record the date of the initial release of the robotic designs. We could therefore not compile a time line that would give us some insights into the development of social robots over time. From a qualitative point of view we were under the impression that older robot design tended to use wheels, and that more recent robots tried to move into the direction of humanoids.

We are also not able to address the question if users would have a preference for legged or wheeled robots. Neither can we address the question what aesthetic implications the use of legs or wheels have.

\section{References}

1. Bartneck, C., Okada, M.: Robotic user interfaces. In: Human and Computer Conference (HC 2001), Aizu, pp. 130-140 (2001)

2. Fong, T., Nourbakhsh, I., Dautenhahn, K.: A survey of socially interactive robots. Robotics and Autonomous Systems 42, 143-166 (2003)

3. Ishiguro, H.: Interactive humanoids and androids as ideal interfaces for humans. In: International Conference on Intelligent User Interfaces (IUI 2006), pp. 2-9. ACM Press, Syndney (2006)

4. JETRO: Trends in the japanese robotics industry. Tech. rep., Japan External Trade Organization(JETRO) (2006)

5. NEC: Papero (2001), http://www.nec.co.jp/products/robot/

6. Sakagami, Y., Watanabe, R., Aoyama, C., Matsunaga, S., Higaki, N., Fujimura, K.: The intelligent asimo: system overview and integration. In: IEEE/RSJ International Conference on Intelligent Robots and Systems, vol. 3, pp. 2478-2483 (2002)

7. Shibata, T., Mitsui, T., Wada, K., Touda, A., Kumasaka, T., Tagami, K., Tanie, K.: Mental commit robot and its application to therapy of children. In: IEEE/ASME International Conference on Advanced Intelligent Mechatronics, vol. 2, pp. 10531058 (2001)

8. United Nations: World Robotics 2008. United Nations Publication, Geneva (2008) 